\title{
TRAVELING WAVE SOLUTIONS OF A GRADIENT SYSTEM: SOLUTIONS WITH A PRESCRIBED WINDING NUMBER. I
}

\author{
DAVID TERMAN
}

\begin{abstract}
Consideration is given to a system of equations of the form $u_{t}=u_{x x}+\nabla F(u), u \in \mathbf{R}^{2}$. In a previous paper [6], conditions of $F$ were given which guarantee that the system possesses infinitely many traveling wave solutions. The solutions are now characterized by how many times they wind around in phase space. A winding number for solutions is defined. It is demonstrated that for each positive integer $K$, there exists at least two traveling wave solutions, each with winding number $K$ or $K+1$.
\end{abstract}

\section{Introduction.}

A. Statement of the problem. Consider the system of reaction-diffusion equations

$$
\begin{aligned}
& u_{1 t}=u_{1 x x}+f_{1}\left(u_{1}, u_{2}\right), \\
& u_{2 t}=u_{2 x x}+f_{2}\left(u_{1}, u_{2}\right)
\end{aligned}
$$

where $u_{1}$ and $u_{2}$ are functions of $(x, t) \in \mathbf{R} \times \mathbf{R}^{+}$. We assume that $f_{1}$ and $f_{2}$ are derived from some potential. That is, there exists a function $F \in C^{2}\left(\mathbf{R}^{2}\right)$ such that

$$
f_{i}\left(u_{1}, u_{2}\right)=\frac{\partial F}{\partial u_{i}}\left(u_{1}, u_{2}\right), \quad i=1,2,
$$

for each $\left(u_{1}, u_{2}\right) \in \mathbf{R}^{2}$. By a traveling wave solution of (1A.1) we mean a nonconstant, bounded solution of the form

$$
\left(u_{1}(x, t), u_{2}(x, t)\right)=\left(U_{1}(z), U_{2}(z)\right), \quad z=x+\theta t .
$$

A traveling wave solution corresponds to a solution which appears to be traveling with constant shape and velocity.

We wish to assume that $F$ looks something like what is shown in Figure 1. Precise assumptions on $F$ will be given shortly. For now we assume that $F$ has at least three local maxima. These are at $\left(u_{1}, u_{2}\right)=A, B$, and $C$ where $F(A)<F(B)<F(C)$. We will be interested in traveling wave solutions which satisfy

$$
\lim _{z \rightarrow-\infty}\left(U_{1}(z), U_{2}(z)\right)=A \text { and } \lim _{z \rightarrow+\infty}\left(U_{1}(z), U_{2}(z)\right)=B .
$$

Motivation for studying this problem is given in $\S 1 \mathrm{E}$.

Note that if $\left(U_{1}(z), U_{2}(z)\right)$ is a traveling wave solution and $\left(V_{1}(z), V_{2}(z)\right)=$ $\left(U_{1}^{\prime}(z), U_{2}^{\prime}(z)\right)$, then $\left(U_{1}, V_{1}, U_{2}, V_{2}\right)$ satisfies the system

$$
\begin{array}{ll}
U_{1}^{\prime}=V_{1}, & V_{1}^{\prime}=\theta V_{1}-F_{U_{1}}\left(U_{1}, U_{2}\right), \\
U_{2}^{\prime}=V_{2}, & V_{2}^{\prime}=\theta V_{2}-F_{U_{2}}\left(U_{1}, U_{2}\right) .
\end{array}
$$

Received by the editors January 16, 1987 and, in revised form, June 8, 1987.

1980 Mathematics Subject Classification (1985 Revision). Primary 35B35, 35K35; Secondary 35B40.

Supported in part by the National Science Foundation under grant \#DMS 8401719 . 


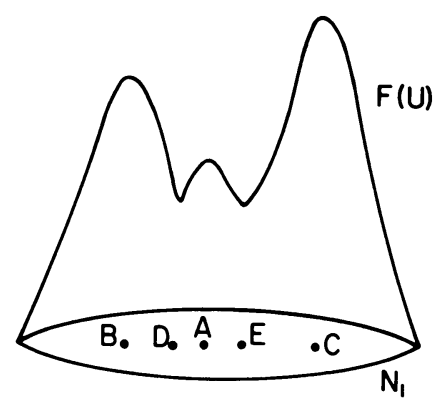

FIGURE 1

We are interested in solutions which satisfy

$$
\lim _{z \rightarrow-\infty}\left(U_{1}, U_{2}, V_{1}, V_{2}\right)=(A, 0) \text { and } \lim _{z \rightarrow+\infty}\left(U_{1}, U_{2}, V_{1}, V_{2}\right)=(B, 0) .
$$

Here, $O=(0,0)$.

In $[6]$ it is proved that under certain assumptions on $F$, which are given shortly, there exists infinitely many traveling wave solutions of (1A.1) which satisfy (1A.3). We now wish to characterize the solutions of (1A.4), (1A.5) by their nodal properties. We shall define a notion of winding number and prove that for each nonnegative integer $K$ there exists a solution of (1A.4), (1A.5) with winding number $K$. The proof of this result is split into two papers. In this paper we reduce the problem of finding a traveling wave solution with a prescribed winding number to a purely algebraic problem. In a later paper [7], we solve the algebraic problem.

B. Assumptions on $F$. The assumptions we make on $F$ are precisely those made in [6]. These assumptions are

(F1) $F \in C^{2}\left(\mathbf{R}^{2}\right)$,

(F2) $F$ has at least three nondegenerate local maxima. These are at $A=$ $\left(A_{1}, A_{2}\right), B=\left(B_{1}, B_{2}\right)$ and $C=\left(C_{1}, C_{2}\right) . \quad F$ has at least two saddles. These are at $D=\left(D_{1}, D_{2}\right)$ and $E=\left(E_{1}, E_{2}\right)$.

(F3) $F(A)<F(B)<F(C)$ and $B_{1}<D_{1}<A_{1}<E_{1}<C_{1}$. Moreover, there exists an $\alpha_{0}$ such that if $\alpha$ is any critical point of $F$ with $\alpha \notin\{A, B, C\}$, then $F(\alpha)<F(A)-\alpha_{0}$. For convenience, we assume that $F(A)=0$ and $A=(0,0)$.

(F4) There exists $W$ such that if $K<W$, then $\{U: F(u) \geq K\}$ is convex.

(F5) If $V_{1}=D_{1}$ or $E_{1}$, then $\partial F_{1} / \partial V_{1}\left(V_{1}, V_{2}\right)=0$ for all $U_{2} \in \mathbf{R}$.

(F6) Let $U=\left(U_{1}, U_{2}\right), V=\left(V_{1}, V_{2}\right)$, and

$$
\begin{aligned}
& N_{1}=\{U: F(U) \geq W\}, \quad X_{1}=\left\{U \in N_{1}: U_{1}<D_{1}\right\}, \\
& X_{2}=\left\{U \in N_{1}: D_{1}<U_{1}<E_{1}\right\}, \quad X_{3}=\left\{U \in N_{1}: E_{1}<U_{1}\right\} .
\end{aligned}
$$

Suppose that $(U(z), V(z))$ is a bounded solution of $(1 \mathrm{~A} .4)$ with $\theta=0$ which satisfies, for $i=1,2$, or 3 ,

(a) $U(z) \in X_{i}$ for all $z \in \mathbf{R}$,

(b) $F(U(z))>F(A)-\alpha_{0}$ for some $z \in \mathbf{R}$,

where $\alpha_{0}$ was defined in (F3). Then $U(z)$ is identically equal to one of the critical points $A, B$, or $C$, and $V(z)=0$ for all $z \in \mathbf{R}$.

Remarks concerning these assumptions were given in [6]. 
C. The winding number. Let

$$
P_{D}=\left\{(U, V): U_{1}=D_{1}, V_{1}=0\right\} \quad \text { and } \quad P_{E}=\left\{(U, V): U_{1}=E_{1}, V_{1}=0\right\} .
$$

Then $P_{D}$ and $P_{E}$ are two dimensional subsets of the four dimensional phase space. We wish to count the number of times solutions of (1A.4), (1A.5) wind around $P_{D}$ and $P_{E}$. Perhaps the most important property of $P_{D}$ and $P_{E}$ is

PROPOSITION 1C.1. $P_{D}$ and $P_{E}$ are invariant with respect to the flow given by (1A.4). That is, if $\left(U_{1}\left(z_{0}\right), U_{2}\left(z_{0}\right), V_{1}\left(z_{0}\right), V_{2}\left(z_{0}\right)\right) \in P_{D}\left(P_{E}\right)$ for some $z_{0}$, then $\left(U_{1}(z), U_{2}(z), V_{1}(z), V_{2}(z)\right) \in P_{D}\left(P_{E}\right)$ for all $z \in \mathbf{R}$.

Proof. From (1A.4) and (F5) we conclude that on $P_{D}$ or $P_{E}, U_{1}^{\prime}=V_{1}=0$ and $V_{1}^{\prime}=\theta V_{1}-F_{U_{1}}\left(U_{1}, U_{2}\right)=0$. These two equalities prove the proposition.

An immediate consequence of this last result is

COROLlary 1C.2. If $(U(z), V(z))$ is a solution of (1A.4), (1A.5), then

$$
(U(z), V(z)) \notin P_{D} \cup P_{E} \quad \text { for all } z .
$$

It now makes sense to count the number of times a solution of (1A.4), (1A.5) winds around $P_{D}$ and $P_{E}$. This is done as follows. Let

$$
\begin{aligned}
& Q_{D}=\left\{(U, V): U_{1}=D_{1}, V_{1}<0, \text { and } U \in N_{1}\right\}, \\
& Q_{E}=\left\{(U, V): U_{1}=E_{1}, V_{1}>0, \text { and } U \in N_{1}\right\} .
\end{aligned}
$$

Definition. Suppose that $(U(z), V(z))$ is a solution of (1A.4), (1A.5). The winding number of $U$ is defined as

$$
h(U)=\operatorname{card}\left\{z:(U(z), V(z)) \in Q_{D} \cup Q_{E}\right\} .
$$

By card $X$ we mean the cardinality of the set $X$.

REMARK 1 . We can also define $h(U)$ if $(U(z), V(z))$ is just a solution of (1A.4) with $\lim _{z \rightarrow-\infty}(U(z), V(z))=(A, 0)$. The definition is, again, (1C.1).

REMARK 2. Our notion of winding number may seem complicated because it involves trajectories in four dimensional phase space. However, one can compute the winding number by just considering $U(z)$ in the two dimensional state space. Recall that $h(U)$ equals the number of times $(U(z), V(z))$ intersects $Q_{D}$ and $Q_{E}$. From the definitions it follows that $\left(U\left(z_{0}\right), V\left(z_{0}\right)\right) \in Q_{D}$ if and only if $U_{1}\left(z_{0}\right)=D_{1}$ and at $z_{0}, U(z)$ crosses

$$
l_{D}=\left\{\left(U_{1}, U_{2}\right): U_{1}=D_{1}\right\}
$$

from right to left. Similarly, $\left(U\left(z_{0}\right), V\left(z_{0}\right)\right) \in Q_{E}$ if and only if $V_{1}\left(z_{0}\right)=E_{1}$ and at $z_{0}, U(z)$ crosses

$$
l_{E}=\left\{\left(U_{1}, U_{2}\right): U_{1}=E_{1}\right\}
$$

from left to right.

D. The main result. Our main result is

THEOREM. Let $K$ be any positive integer. Then there exists a traveling wave solution $U(z)$ of (1A.1), (1A.3) such that either $h(U)=K$ or $h(U)=K+1$.

As we mentioned earlier this theorem is proved in two parts. In this paper we reduce the proof of the theorem to a purely algebraic problem. In [7] we solve the algebraic problem. 
REMARK 1. The fact that we have either $h(U)=K$ or $h(U)=K+1$ may be disturbing, because one would expect there to exist a traveling wave solution such that $h(U)=K$. The reason that we obtain the weaker result is that we are counting the number of times the solution winds around two objects, namely $P_{D}$ and $P_{E}$.

REMARK 2 . We actually prove that for each integer $K$ there exists at least two traveling wave solutions, each with winding number $K$ or $K+1$.

E. Motivation and outline of the proof. In this paper we extend the results of many previous papers. In [1, p. 73], Conley proved the existence of at least one traveling wave solution for a certain class of gradient systems. Conley's proof was based on his generalization of Morse theory. The techniques developed in this paper are an extension, or refinement, of the Conley index. Another extension of Conley's theory is the connection matrix, see [2, 3, and 4]. The connection matrix has been used by Mischaikow [3] and Reinick [4] to prove the existence of traveling wave solutions for certain gradient systems. While the connection matrix is a beautiful theory which applies to very general problems, it does not contain the refined information needed about a given flow to obtain the results of this paper; that is, to characterize the solutions by a winding number.

There are also some technical problems in applying the connection matrix to the problem we consider. For example, we do not assume that the set of wave speeds $\theta$ for which there exists a traveling wave solution is discrete. It is conceivable that for every $\theta>0$ there exists a traveling wave solution with speed $\theta$, but the set of connecting orbits in phase space is topologically small. In such a situation there are uncountably many traveling wave solutions, but it is more difficult to prove that for each integer $K$, there exists a traveling wave solution with winding number $K$.

In $[8,9]$ we use the techniques developed in this paper to prove the existence of infinitely many radial solutions of elliptic equations of the form

$$
\Delta u_{1}+f_{1}\left(u_{1}, u_{2}\right)=0, \quad \Delta u_{2}+f_{2}\left(u_{1}, u_{2}\right)=0
$$

where $\left(f_{1}, f_{2}\right)$ satisfy $(1 \mathrm{~A} .2)$ and $F\left(u_{1}, u_{2}\right)$ satisfies (F1)-(F6). We also prove that for each positive integer $K$, there exists a radial solution with winding number $K$ or $K+1$. We reduce the proof of that result to the same algebraic problems obtained in this paper. The radial solution problem is technically more difficult than the traveling wave problem. The geometrical construction given in this paper is also more straightforward than that given in $[8,9]$.

This paper generalizes many of the results in [5] where we prove the existence of traveling wave solutions for the scalar equation $u_{t}=u_{x x}+f(u)$. Very few assumptions are made on $f(u)$. In order to motivate the proof given in this paper, we shall briefly discuss the main ideas developed in [5].

A traveling wave solution of the scalar equation satisfies the system of ordinary differential equations

$$
u^{\prime}=v, \quad v^{\prime}=\theta v-f(u) .
$$

For each $\theta$ for which there does not exist a traveling wave solution we assign an algebraic object, which we denote by $A(\theta)$. In [5], $A(\theta)$ was an array of positive integers. It had the property that if $\theta_{0}<\theta_{1}$ and $A\left(\theta_{0}\right) \neq A\left(\theta_{1}\right)$, then there must exist a traveling wave solution for some $\theta \in\left(\theta_{0}, \theta_{1}\right)$. In order to prove the existence of infinitely many traveling wave solutions we needed to compute three things. 
These are

(a) $A\left(\theta^{0}\right)$ for $\theta^{0}$ very large,

(b) some property of $A\left(\theta_{0}\right)$ for $0<\theta_{0} \ll 1$,

(c) a formula which relates $A\left(\theta_{1}+\delta\right)$ with $A\left(\theta_{1}-\delta\right)$.

Here we assume that there exists a traveling wave solution with speed $\theta_{1}$ and there does not exist any traveling wave solution with speed $\theta$ if $0<\left|\theta-\theta_{1}\right| \leq \delta$.

The steps in proving the existence of infinitely many traveling wave solutions are the following. First we use (1E.2a) and (1E.2b) to show that $A\left(\theta^{0}\right) \neq A\left(\theta_{0}\right)$. This implies that there exist at least one traveling wave solution. Let $\theta_{1}=$ $\sup \{\theta$ : there exists a traveling wave solution with speed $\theta\}$. Assume that for some $\delta>0$, there are no traveling wave solution with speed $\theta$ such that $0<\left|\theta-\theta_{1}\right| \leq \delta$. Then $A\left(\theta_{1}+\delta\right)=A\left(\theta^{0}\right)$. Using (1E.2c) we compute $A\left(\theta_{1}-\delta\right)$. If $A\left(\theta_{1}-\delta\right) \neq A\left(\theta_{0}\right)$, then there must exist another traveling wave solution with speed $\theta_{2} \in\left(\theta_{0}, \theta_{1}-\delta\right)$. Continuing this process, we were able to show that in many cases there does exist infinitely many traveling wave solutions.

We point out that, in general, one cannot compute $A\left(\theta_{0}\right)$ explicitly for small $\theta_{0}$. This is because if there does exist infinitely many traveling wave solutions, then their wave speeds will converge to $\theta=0$. Hence, $A(\theta)$ will change infinitely often as $\theta \rightarrow 0$.

The program just outlined is carried out in this paper for the system of equations (1A.1). For $A(\theta)$ we use an element of the free group on four elements. The formula for $A\left(\theta^{0}\right), \theta^{0} \gg 1$, is given in $\S 4 \mathrm{~B}$. The property of $A\left(\theta_{0}\right), 0<\theta_{0} \ll 1$, used is given in Proposition 2.5. Actually, Proposition 2.5 is a geometric statement about the winding number of trajectories in phase space for $\theta$ small. This geometric statement is converted into an algebraic statement in [6, Proposition 5A.1] and [7, Proposition 1E.1]. Finally, the formula for how $A(\theta)$ changes after a solution is given in Proposition 4D.1.

The major technical difficulty in applying this program to systems is that the set of wave speeds may not be discrete. Hence, the algebraic invariant cannot depend on just the wave speeds $\theta$. Hopefully, this will help motivate some of the later constructions, particularly Proposition 3.4.

What we have discussed so far is just used to prove the existence of infinitely many traveling wave solutions. To actually characterize the solutions by a winding number, we need a formula which relates the winding number of a solution with the algebraic invariants. This formula is derived in $\S 4 \mathrm{E}$.

In this paper we use the notation and results presented in [6]. This material is reviewed in $\S 2$.

F. The algebraic problem. In this section we simply state the algebraic problem which we reduce the proof of the theorem to.

Let $F_{4}$ be the set of words on the four elements $\{\alpha, \beta, \gamma, \delta\}$. That is, if $\Gamma \in F_{4}$, then we can express $\Gamma$ as

$$
\Gamma=\lambda_{1}^{e_{1}} \lambda_{2}^{e_{2}} \cdots \lambda_{K}^{e_{K}}
$$

where, for such $i, \lambda_{i} \in\{\alpha, \beta, \gamma, \delta\}$ and $e_{i} \in\{-1,1\}$.

For $\Gamma \in F_{4}$, let $\Gamma^{*}$ equal the subset of $F_{4}$ of all elements which upon cancellations equal $\Gamma$. For example, if $\Gamma=\alpha \beta$, then $\alpha^{2} \beta \beta^{-1} \alpha^{-1} \beta \in \Gamma^{*}$. 
If $\Gamma \in F_{4}$ is given by (1E.1), let

$$
\omega(\Gamma)=\sum_{i=1}^{K} e_{i}
$$

and

$$
|\Gamma|=\sup _{1 \leq J \leq K} \sum_{i=1}^{J} e_{i} .
$$

Actually, to state the algebraic problem we must define another integer $\|\Gamma\|$ for $\Gamma \in F_{4}$. We are not able to define this now because it is necessary to develop quite a bit of the algebraic theory first. For now, we assume that $\|\Gamma\|$ is well defined. It will be defined in [7, Formula 2.7]. We point out that if $\Gamma \in F_{4}$, then $\|\Gamma\| \leq|\Gamma|$.

We now state the algebraic problem.

Proposition 1F.1. Let $\left\{\Gamma_{k}\right\}, k=1,2, \ldots$, be an infinite sequence of elements of $F_{4}$ which satisfy

(a) $\Gamma_{1}=\beta \gamma^{-1}$, $M$,

(b) for each positive integer $M$ there exists $K$ such that if $k>K$, then $\left\|\Gamma_{k}\right\|>$

(c) for each $k$ there exists $\Gamma_{A}, \Gamma_{B} \in F_{4}$ and an integer $r$ such that $\Gamma_{A} \Gamma_{B} \in \Gamma_{k}^{*}$ and $\Gamma_{A}\left(\alpha \beta \gamma^{-1} \delta^{-1}\right)^{r} \Gamma_{B} \in \Gamma_{k+1}^{*}$.

Let $h_{k}=\omega\left(\Gamma_{A}\right)$. Then for each positive integer $K$ there exists $k$ such that either $h_{k}=K$ or $h_{k}=K+1$.

REMARK. In (c), by $\Gamma_{A} \Gamma_{B}$ we mean the following. If

$$
\Gamma_{A}=\lambda_{1}^{e_{1}} \lambda_{2}^{e_{2}} \cdots \lambda_{K}^{e_{K}} \quad \text { and } \quad \Gamma_{B}=\eta_{1}^{f_{1}} \eta_{2}^{f_{2}} \cdots \eta_{J}^{f_{J}}
$$

then

$$
\Gamma_{A} \Gamma_{B}=\lambda_{1}^{e_{1}} \cdots \lambda_{K}^{e_{K}} \eta_{1}^{f_{1}} \cdots \eta_{J}^{f_{J}}
$$

2. Summary of the results in [6]. In this section we recall the results needed from [6]. We do not prove the results here.

Recall the sets $N_{1}, P_{D}$ and $P_{E}$ defined in the previous section. Let

$$
N_{2}=\left\{(U, V): U \in N_{1},\|V\| \leq \bar{V}\right\}
$$

where $\bar{V}$ is a large constant to be determined. Let $N=N_{2} \backslash\left(P_{D} \cup P_{E}\right)$. In [6] we proved that if $\bar{V}$ is sufficiently large, then all bounded solutions of (1A.4) lie in $N_{2}$. Together with Corollary 1C.2, this implies that all solutions of (1A.4), (1A.5) lie in $N$.

In [6] we proved

LEMMA 2.1. There exists $T$ such that no solutions of (1A.4), (1A.5) exist for $\theta \geq T$.

Let

$$
H(U, V)=\|V\|^{2} / 2+F(U) .
$$

On a solution, $(U(z), V(z))$, let $H(z)=H(U(z), V(z))$. One easily checks that on a solution,

$$
H^{\prime}(z)=\theta\|V\|^{2}
$$




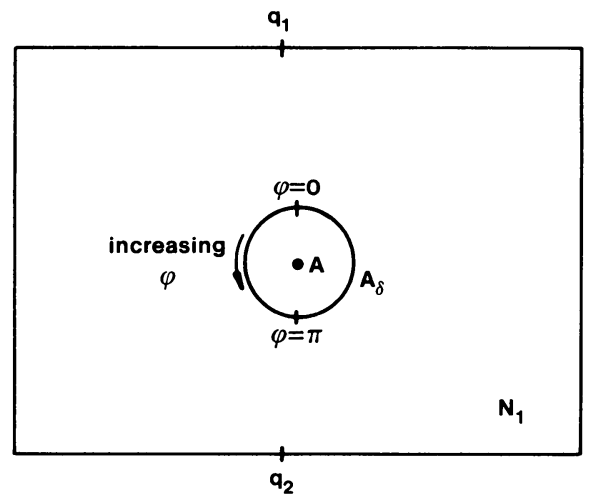

Figure 2

Hence, $H(z)$ is increasing on a solution if $\theta>0$. If $(U(z), V(z))$ satisfies (1A.5), then

$$
\lim _{z \rightarrow-\infty} H(z)=F(A) \text { and } \lim _{z \rightarrow+\infty} H(z)=F(B) .
$$

One consequence is that on a solution of (1A.4), (1A.5), we have $H(z)<F(B)$. This implies that there exists $\delta>0$ such that if $(U(z), V(z))$ is a solution of (1A.4), (1A.5), then for all $z$,

$$
U(z) \notin C_{\delta} \equiv\{U:\|U-C\| \leq \delta\} .
$$

Hence the values of $F(U)$ in $C_{\delta}$ do not matter if we are only interested in solutions of (1A.4), (1A.5). In particular, $F(U)$ may be assumed to be arbitrarily large in $C_{\delta}$. In [6] we showed that we can choose $F(U)$ to be so large in $C_{\delta}$ that if $\theta<T$ and $\lim _{z \rightarrow-\infty}(U(z), V(z))=(A, 0)$, then $U(z) \neq C$ for all $z$, and $\lim _{z \rightarrow \infty} U(z) \neq C$.

In [6] we then studied

$$
W_{A}^{\theta}=\text { unstable manifold at }(A, 0) \text { for a particular } \theta \text {. }
$$

We showed that for each $\theta, \operatorname{dim} W_{A}^{\theta}=2$. Moreover, from the Stable Manifold Theorem,

Proposition 2.2. Near $(A, 0), W_{A}^{\theta}$ is a $C^{2}$ injectively immersed, two dimensional manifold. Moreover, the tangent space to $W_{A}^{\theta}$ at $(A, O)$ is the linear subspace spanned by the two eigenvectors corresponding to the positive eigenvalues of the linearized equations at $(A, O)$.

An important consequence of this proposition is

Proposition 2.3. There exists $\delta>0$ such that if $A_{\delta}=\{U:\|A-U\|=\delta\}$, then for each $\theta$,

(a) for each $U_{0} \in A_{\delta}$, there exists a unique $V_{0} \in \mathbf{R}^{2}$ such that $\left(U_{0}, V_{0}\right) \in W_{A}^{\theta}$,

(b) if $(U(z), V(z))$ is any nontrivial trajectory in $W_{A}^{\theta}$, then there exists a unique $z_{0}$ such that $U\left(z_{0}\right) \in A_{\delta}$.

This proposition implies that for each $\theta$ we may parametrize the nontrivial trajectories in $W_{A}^{\theta}$ by the points in $A_{\delta}$. Let us parametrize the points in $A_{\delta}$ by the angle $\varphi$ as shown in Figure 2 . 
Let

$$
D_{1}=\{(\varphi, \theta): 0 \leq \varphi \leq 2 \pi, 0<\theta \leq T\} .
$$

To each $(\varphi, \theta) \in D_{1}$, there corresponds a unique trajectory in $W_{A}^{\theta}$. If $(\theta, \varphi)=d$, then we denote this trajectory by $\gamma(d)(z)=(U(d)(z), V(d)(z))$. Here, $z$ is the independent variable along the trajectory. For $d \in D_{1}$, let $h(d)=h(U(d))$ where $h(U(d))$ was defined in $\S 1 \mathrm{C}$.

Crucial to the proof of the theorem is the following result proved in [1].

Proposition 2.5. Given $M$, there exists $\theta_{M}$ such that if $0<\theta<\theta_{M}, 0 \leq$ $\varphi \leq 2 \pi, d=(\varphi, \theta)$ and $U(d)(z)=B$ for some $z$, then $h(d)>M$.

The next step in [6] was to define an algebraic invariant. To do this we first need

LEMMA 2.6. Fix $\theta \in[0, T]$ and $q \in \partial X_{2}$ where $X_{2}$ was defined in (1B.1). Then there exists $\varphi=\varphi(\theta, q)$ such that $U(\varphi(\theta, q), \theta)\left(z_{0}\right)=q$ for some $z_{0}$, and $U(\varphi(\theta, q), \theta)(z) \in X_{2}$ for $z<z_{0}$. Moreover, $\varphi(\theta, q)$ can be chosen to depend continuously on $\theta$ and $q$.

Let $q_{1}$ and $q_{2}$ be any points of $\partial X_{2}$ as shown in Figure 2. That is, $q_{1}$ is on the top side of $N_{1}$ and $q_{2}$ is on the bottom side. Choose continuous functions $\varphi_{1}(\theta)$ and $\varphi_{2}(\theta)$ such that for $i=1,2, U\left(\varphi_{i}(\theta), \theta\right)(z)$ leaves $X_{2}$ through $q_{i}$. We assume, without loss of generality, that $\varphi_{1}(\theta)<\varphi_{2}(\theta)$ for each $\theta$.

Let

$$
\begin{aligned}
& D=\left\{(\varphi, \theta) \in D_{1}: \varphi_{1}(\theta) \leq \varphi \leq \varphi_{2}(\theta)\right\} \\
& X=\{d \in D: \gamma(d)(z) \rightarrow(B, 0) \text { as } z \rightarrow \infty\}, \\
& Y=D \backslash X
\end{aligned}
$$

Then $X$ corresponds to solutions of (1A.4), (1A.5). We are trying to prove that for each $K$ there exists $x \in X$ such that either $h(x)=K$ or $h(x)=K+1$.

Suppose that $y \in Y$. We claim that $\gamma(y)(z)$ must leave $N$. Because $H(z)$ is increasing on solutions, if $\gamma(y)(z)$ does not leave $N$, then either $\lim _{z \rightarrow \infty} \gamma(d)(z)=$ $(B, 0)$ or $\lim _{z \rightarrow \infty} \gamma(d)(z)=(C, 0)$. By the definition of $Y$, we cannot have $\lim _{z \rightarrow \infty} \gamma(d)(z)=(B, 0)$. Moreover, we chose $F(C)$ so large that if $d \in D$, then $U(d)(z) \neq C$ for all $z$ and $\lim _{z \rightarrow \infty} U(d)(z) \neq C$. Hence, $\gamma(d)(z)$ must leave $N$.

Let

$$
\mathcal{E}=\left\{(U, V) \in \partial N \backslash\left(P_{D} \cup P_{E}\right):\|V\|<\bar{V}\right\} .
$$

In [6] we showed that $\bar{V}$ can be chosen so large that if $y \in Y$, then $\gamma(y)(z)$ must leave $N$ through $\mathcal{E}$. Hence, we have a mapping $\Lambda: Y \rightarrow \mathcal{E}$ defined by $\Lambda(y)$ equals the place where $\gamma(y)(z)$ leaves $N$. In [1] we showed that $\Lambda$ is continuous.

Let $I$ be the unit interval and $\mathcal{G}$ the set of functions $g: I \rightarrow Y$ such that

(a) $g$ is continuous,

(b) $g(0) \in\left\{(\varphi, \theta): \varphi=\varphi_{1}(\theta)\right\}$,

(c) $g(1) \in\left\{(\varphi, \theta): \varphi=\varphi_{2}(\theta)\right\}$.

If $g \in \mathcal{G}$, then we have a continuous map $\Lambda \circ g: I \rightarrow \mathcal{E}$.

Note that $\mathcal{E}$ is topologically an annulus with four holes removed. We now define two algebraic objects, $\Gamma^{*}(g)$ and $\Gamma(g)$, which indicate how the curve $(\Lambda \circ g)(I)$ winds around the four holes. They will be elements of $F_{4}$, the set of words on the four elements $\alpha, \beta, \gamma$, and $\delta$. 


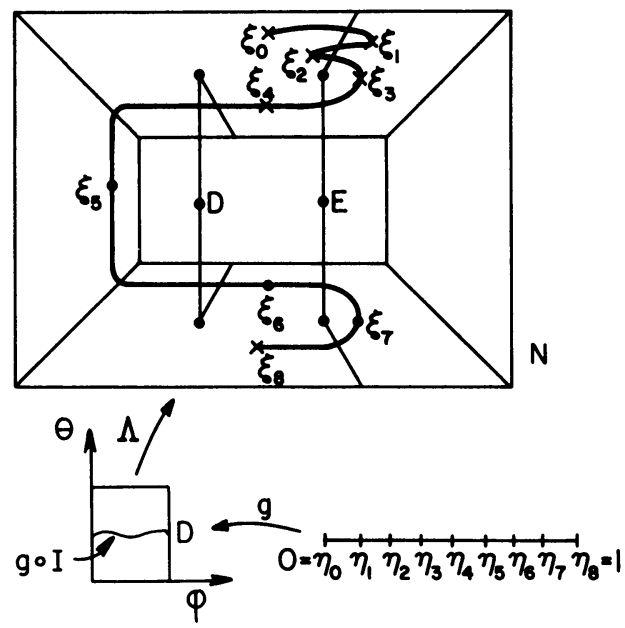

FIGURE 3

We begin with some notation. For convenience we assume that $N_{1}$ is the square

$$
N_{1}=\left\{\left(U_{1}, U_{2}\right):\left|U_{1}\right| \leq W,\left|U_{2}\right| \leq W\right\} .
$$

Let

$$
\begin{aligned}
& \mathcal{E}_{1}=\left\{(U, V) \in \mathcal{E}: U_{1}>E_{1}\right\}, \\
& \mathcal{E}_{2}=\left\{(U, V) \in \mathcal{E}: D_{1}<U_{1}<E_{1}, U_{2}=W\right\}, \\
& \mathcal{E}_{3}=\left\{(U, V) \in \mathcal{E}: U_{1}<D_{1}\right\}, \\
& \mathcal{E}_{4}=\left\{(U, V) \in \mathcal{E}: D_{1}<U_{1}<E_{1}, U_{2}=-W\right\}, \\
& l_{1}=l_{\alpha}^{+}=\left\{(U, V): U_{1}=E_{1}, U_{2}=W, 0<V_{1} \leq \bar{V}\right\}, \\
& l_{2}=l_{\alpha}^{-}=\left\{(U, V): U_{1}=E_{1}, U_{2}=W,-\bar{V} \leq V_{1}<0\right\}, \\
& l_{3}=l_{\beta}^{+}=\left\{(U, V): U_{1}=D_{1}, U_{2}=W, 0<V_{1} \leq \bar{V}\right\}, \\
& l_{4}=l_{\beta}^{-}=\left\{(U, V): U_{1}=D_{1}, U_{2}=W,-\bar{V} \leq V_{1}<0\right\}, \\
& l_{5}=l_{\gamma}^{+}=\left\{(U, V): U_{1}=D_{1}, U_{2}=-W, 0<V_{1} \leq \bar{V}\right\}, \\
& l_{6}=l_{\gamma}^{-}=\left\{(U, V): U_{1}=D_{1}, U_{2}=-W,-\bar{V} \leq V_{1}<0\right\}, \\
& l_{7}=l_{\delta}^{+}=\left\{(U, V): U_{1}=E_{1}, U_{2}=-W, 0<V_{1} \leq \bar{V}\right\}, \\
& l_{8}=l_{\delta}^{-}=\left\{(U, V): U_{1}=E_{1}, U_{2}=-W,-\bar{V} \leq V_{1}<0\right\} .
\end{aligned}
$$

Assume that $g \in \mathcal{G}$, choose $\eta_{k} \in[0,1], k=1,2, \ldots, K$, such that

(a) $\eta_{1}=0, \eta_{K}=1$,

(b) $\eta_{k}<\eta_{k+1}$ for all $k$,

(c) $(\Lambda \circ g)\left(\eta_{k}\right) \in \bigcup_{i=1}^{4} \mathcal{E}_{i}$ for all $k$,

(d) $(\Lambda \circ g)\left(\eta_{k}, \eta_{k+1}\right)$ intersects at most one of the line segments $l_{i}, i=1, \ldots, 8$, for each $k$.

We refer to $\eta^{*}=\left\{\eta_{1}, \ldots, \eta_{K}\right\}$ as a $g$-partition. It is not hard to prove that a $g$-partition does exist. 
To define $\Gamma^{*}\left(g, \eta^{*}\right)$, we must first define

$$
\lambda_{k}=\lambda\left(\eta_{k}\right) \in\{\alpha, \beta, \gamma, \delta\} \quad \text { and } \quad e_{k}=e\left(\eta_{k}\right) \in\{-1,0,1\} .
$$

These quantities are determined by the following table. In Table 1, we let, for $s \in$ $[0,1], \Phi(s) \equiv(\Lambda \circ g)(s)$. For each case not shown in the chart we let $e_{k}=e\left(\eta_{k}\right)=0$. For this case we do not define $\lambda_{k}=\lambda\left(\eta_{k}\right)$ because, as we shall see, since $e_{k}=0$ the choice of $\lambda_{k}$ does not matter. Then define

$$
\Gamma^{*}\left(g, \eta^{*}\right)=\prod_{i=1}^{K} \lambda_{i}^{e_{i}} \stackrel{\text { def }}{=} \lambda_{1}^{e_{1}} \lambda_{2}^{e_{2}} \cdots \lambda_{K}^{e_{K}} .
$$

TABLE 1

\begin{tabular}{ccccr}
\hline$\Phi\left(\eta_{k}\right) \in$ & $\Phi\left(\eta_{k+1}\right) \in$ & $\begin{array}{c}\Phi(\eta) \text { crosses } \\
\text { for } \eta \in\left(\eta_{k}, \eta_{k+1}\right)\end{array}$ & $\lambda\left(\eta_{k+1}\right)$ & $e\left(\eta_{k+1}\right)$ \\
\hline $\mathcal{E}_{2}$ & $\mathcal{E}_{1}$ & $l_{\alpha}^{+}$ & $\alpha$ & 1 \\
$\mathcal{E}_{1}$ & $\mathcal{E}_{2}$ & $l_{\alpha}^{+}$ & $\alpha$ & -1 \\
$\mathcal{E}_{2}$ & $\mathcal{E}_{3}$ & $l_{\beta}^{-}$ & $\beta$ & 1 \\
$\mathcal{E}_{3}$ & $\varepsilon_{2}$ & $l_{\beta}^{-}$ & $\beta$ & -1 \\
$\mathcal{E}_{3}$ & $\mathcal{E}_{4}$ & $l_{\gamma}^{-}$ & $\gamma$ & -1 \\
$\mathcal{E}_{4}$ & $\mathcal{E}_{3}$ & $l_{\gamma}^{-}$ & $\gamma$ & 1 \\
$\mathcal{E}_{4}$ & $\mathcal{E}_{1}$ & $l_{\delta}^{+}$ & $\delta$ & 1 \\
$\mathcal{E}_{1}$ & $\mathcal{E}_{4}$ & $l_{\delta}^{+}$ & $\delta$ & -1
\end{tabular}

Let $\Gamma(g)$ equal $\Gamma^{*}\left(g, \eta^{*}\right)$ with all cancellations. In [6] we showed that $\Gamma(g)$ does not depend on the $g$-partition $\eta^{*}$. Let $\Gamma^{*}(g)$ be the subset of $F_{4}$ consisting of all elements, which yield, after all cancellations, $\Gamma(g)$. Figure 3 is an example. In the figure, $\xi_{k}=\Phi\left(\eta_{k}\right)$. Then

$$
\Gamma^{*}\left(g, \eta^{*}\right)=\alpha \alpha^{-1} \alpha \beta \gamma^{-1} \delta^{-1} \text { and } \Gamma(g)=\alpha \beta \gamma^{-1} \delta^{-1} .
$$

Recall that for each $d=(\varphi, \theta) \in D$ there corresponds a trajectory $\gamma(d)(z) \in W_{A}^{\theta}$. Moreover there is a winding number, $h(d)$, which counts the number of times $\gamma(d)(z)$ winds around $P_{D}$ and $P_{E}$. Suppose tht $g(s) \in \mathcal{G}$ and $s_{0} \in I$. In [1] we derived a formula for $h_{1}\left(s_{0}\right) \equiv h\left(g\left(s_{0}\right)\right)$ in terms of $\Gamma^{*}\left(g, \eta^{*}\right)$ for some $g$-partition $\eta^{*}$. To describe this result we need some notation.

Suppose that $\Gamma \in F_{4}$ is given by $\Gamma=\lambda_{1}^{e_{1}} \cdots \lambda_{J}^{e_{J}}$ where each $\lambda_{i} \in\{\alpha, \beta, \gamma, \delta\}$ and $e_{i} \in\{-1,1\}$. Let

$$
\omega(\Gamma)=\sum_{i=1}^{J} e_{i}
$$

Let $g \in \mathcal{G}$ and $\eta^{*}$ be a $g$-partition. Define the map $\Lambda\left(g, \eta^{*}\right): I \rightarrow F_{4}$ as follows. Suppose that $\eta^{*}=\left\{\eta_{1}, \ldots, \eta_{K}\right\}$ and $\eta_{k} \leq s<\eta_{k+1}$. Then define

$$
\Lambda\left(g, \eta^{*}\right)(s)=\prod_{i=1}^{k} \lambda_{i}^{e_{i}}=\lambda_{1}^{e_{1}} \cdots \lambda_{k}^{e_{k}} .
$$

The $\lambda_{i}$ and $e_{i}$ are defined as in (2.3) and Table 1. Finally, define $\Lambda_{1}\left(g, \eta^{*}\right): I \rightarrow Z^{+}$, where $Z^{+}$is the set of nonnegative integers, by

$$
\Lambda_{1}\left(g, \eta^{*}\right)(s)=\left[\omega \circ \Lambda\left(g, \eta^{*}\right)\right](s) .
$$


In [6], we prove

PROPOSITION 2.6. Assume that $g \in \mathcal{G}, \eta^{*}=\left\{\eta_{1}, \ldots, \eta_{K}\right\}$ is a g-partition, and $\eta_{k} \leq s_{0}<\eta_{k+1}$. Then either

$$
h_{1}\left(s_{0}\right)=\Lambda_{1}\left(g, \eta^{*}\right)\left(\eta_{k}\right) \quad \text { or } \quad h_{1}\left(s_{0}\right)=\Lambda_{1}\left(g, \eta^{*}\right)\left(\eta_{k+1}\right) .
$$

3. Properties of $X$. In this section we prove some important properties of the set $X$ which, as we recall, are the points in $D$ which correspond to solutions of (1A.4), (1A.5). One cannot expect $X$ to be too "large" because for each $\theta$, the dimension of the unstable manifold at $(A, 0)$ is two and the dimension of the stable manifold $(B, O)$ is two. One does not expect two, two dimensional manifolds to intersect very often in four dimensional phase space. On the other hand, there is a lot of room in $\mathbf{R}^{4}$, so the set of connecting orbits may be infinite, but still topologically small. This may not seem like much of a problem, because we would like to have an infinite number of solutions, however we are also trying to prove the existence of a solution with a prescribed winding number. The proof of this last result becomes more difficult if the set of solutions is too bizarre.

LEMMA 3.1. Given $x_{0} \in X$ there exists $\delta\left(x_{0}\right)$ such that if $x \in X$ and $\left\|x-x_{0}\right\|<$ $\delta\left(x_{0}\right)$, then $h(x)=h\left(x_{0}\right)$.

PrOOF. Let

$$
\begin{aligned}
S & =\left\{U: F(U) \geq \frac{1}{2} F(D)\right\} \cap X_{1}, \\
d & =\sup \left\{U_{1}:\left(U_{1}, U_{2}\right) \in S\right\}, \\
d_{0} & =D_{1}-d, \\
B_{\gamma} & =\{U:\|B-U\| \leq \gamma\} \quad \text { for } \gamma>0 .
\end{aligned}
$$

Assume that $x_{0}=\left(\varphi_{0}, \theta_{0}\right)$. Choose $\gamma>0$ so that

$$
\gamma<\frac{1}{2}\left(B_{1}+d\right), \quad B_{\gamma} \subset S,
$$

and in $B_{\gamma}$,

$$
F(U)>F(B)-\left(d_{0} / \bar{V}\right)\left(\frac{1}{2} \theta_{0}\right)|F(D)| .
$$

Now $U\left(x_{0}\right)(z)$ crosses $l_{D}$ and $l_{E} h\left(x_{0}\right)$ times, enters $B_{\gamma}$ and then approaches $B$ as $z \rightarrow \infty$. By continuous dependence there exists $\delta_{1}$ such that if $\left\|x_{1}-x_{0}\right\|<$ $\delta_{1}$, then $U\left(x_{1}\right)(z)$ crosses $l_{D}$ and $l_{E} h\left(x_{0}\right)$ times and then enters $B_{\gamma}$. Hence, if $\left\|x_{1}-x_{0}\right\|<\delta_{1}$, then $h\left(x_{1}\right) \geq h\left(x_{0}\right)$. So suppose $\left\|x_{1}-x_{0}\right\|<\delta_{1}$ and $h\left(x_{1}\right)>h\left(x_{0}\right)$. Let $x_{1}=\left(\varphi_{1}, \theta_{1}\right)$. We assume that $\delta_{1}$ is so small that $\theta_{1}>\frac{1}{2} \theta_{0}$.

Because $h\left(x_{1}\right)>h\left(x_{0}\right)$, after $U\left(x_{1}\right)(z)$ crosses $l_{D}$ and $l_{E} h\left(x_{0}\right)$ times and enters $B_{\gamma}$ it must leave $B_{\gamma}$ and cross $l_{D}$ again. Suppose that $U\left(x_{1}\right)(z)$ leaves $B_{\gamma}$ at $z=z_{1}$ and then crosses $l_{D}$ at $z=z_{2}$. We wish to estimate the change in $H(z)$ from $z=z_{1}$ to $z=z_{2}$. Recall that

$$
H^{\prime}(z)=\theta_{1}\|V(z)\|^{2}>\left(\theta_{0} / 2\right)\|V(z)\|^{2} .
$$

Because $B_{\gamma} \subset S$ we can choose $z_{3} \in\left(z_{1}, z_{2}\right)$ such that $U\left(x_{1}\right)(z)$ leaves $S$ at $z_{3}$. For $z \in\left(z_{3}, z_{2}\right)$

$$
F(U)<\frac{1}{2} F(D)=-\frac{1}{2}|F(D)|
$$


since, by assumption, $F(D)<F(A)=0$. Now, because $H(z)>F(A)=0$,

$$
H(z)=\|V(z)\|^{2} / 2+F(U(z))>0 .
$$

Therefore,

$$
\|V(z)\|^{2}>|F(D)| \text { for } z \in\left(z_{3}, z_{2}\right) .
$$

We now estimate $z_{2}-z_{3}$. Since $U_{1}\left(x_{1}\right)\left(z_{2}\right)=D_{1}$ and $U_{1}\left(x_{1}\right)\left(z_{3}\right) \leq d$ it follows that

$$
U_{1}\left(x_{1}\right)\left(z_{2}\right)-U_{1}\left(x_{1}\right)\left(z_{3}\right) \geq d_{0} .
$$

Moreover, as long as $\gamma\left(x_{1}\right)(z) \in N$, we have $\|V(z)\|<\bar{V}$. It then follows that

$$
z_{2}-z_{3}>d_{0} / \bar{V} \text {. }
$$

Therefore, from (3.2)-(3.4),

$$
H\left(z_{2}\right)-H\left(z_{3}\right)=\int_{z_{3}}^{z_{2}} H^{\prime}(z) d z>\frac{\theta_{0} d_{0}}{2 \bar{V}}|F(D)| .
$$

Let $z_{4}=\sup \left\{z: U\left(x_{1}\right)(z) \in B_{\gamma}\right\}$. Clearly, $z_{4}>z_{2}$. Then from (3.1) and (3.5),

$$
\begin{aligned}
H\left(z_{4}\right) & =H\left(z_{1}\right)+H\left(z_{4}\right)-H\left(z_{1}\right) \geq H\left(z_{1}\right)+\left[H\left(z_{2}\right)-H\left(z_{3}\right)\right] \\
& >F(B)-\frac{d_{0} \theta_{0}}{2 \bar{v}}|F(D)|+\frac{d_{0} \theta_{0}}{2 \bar{v}}|F(D)|>F(B) .
\end{aligned}
$$

This is impossible because $\lim _{z \rightarrow \infty} \gamma\left(x_{1}\right)(z)=(B, 0)$ implies that $H(z)<F(B)$ for all $z$.

LEMMA 3.2. $X$ is a closed subset of $D$.

ProOF. This is equivalent to showing that $Y$ is an open subset of $D$. However, if $y_{0} \in Y$, then $\gamma\left(y_{0}\right)(z)$ leaves $N$. By continuous dependence of solutions, all trajectories near $\gamma\left(y_{0}\right)(z)$ leave $N$. In particular, there exists $\delta$ such that if $\left\|y-y_{0}\right\|<\delta$, then $\gamma(y)(z)$ leaves $N$. Therefore, $Y$ is open.

COROLlary 3.3. Fix $k>0$ and let $Z_{k}=\{x \in X: h(x)=k\}$. Then $Z_{k}$ is compact.

PrOOF. From the previous two results we know that $Z_{k}$ is closed. Proposition 2.5 implies that there exists $\theta_{0}>0$ such that if $x=(\varphi, \theta) \in Z_{k}$, then $\theta>\theta_{0}$. This proves the result.

We now prove the most important result of this section.

Proposition 3.4. $X$ can be written as a countable, disjoint union, $X=X_{1} \cup$ $X_{2} \cup \cdots$, such that for each $k$,

(a) $X_{k}$ is a compact subset of $D$,

(b) if $x_{1}, x_{2} \in X_{k}$, then $h\left(x_{1}\right)=h\left(x_{2}\right)$,

(c) there exist mutually disjoint, closed curves $C_{k} \subset Y$ such that

(i) $X_{k}$ lies in the disc bounded by $C_{k}$,

(ii) for each $y \in C_{k}$, there exists $x \in X_{k}$ with $\|y-x\|<\delta_{k}$.

REMARKS. (1) We do not claim that if $x \in X_{k}$, then $h(x)=k$.

(2) It is possible that there exists $i \neq j$ such that if $x_{1} \in X_{i}$ and $x_{2} \in X_{j}$, then $h\left(x_{1}\right)=h\left(x_{2}\right)$. 


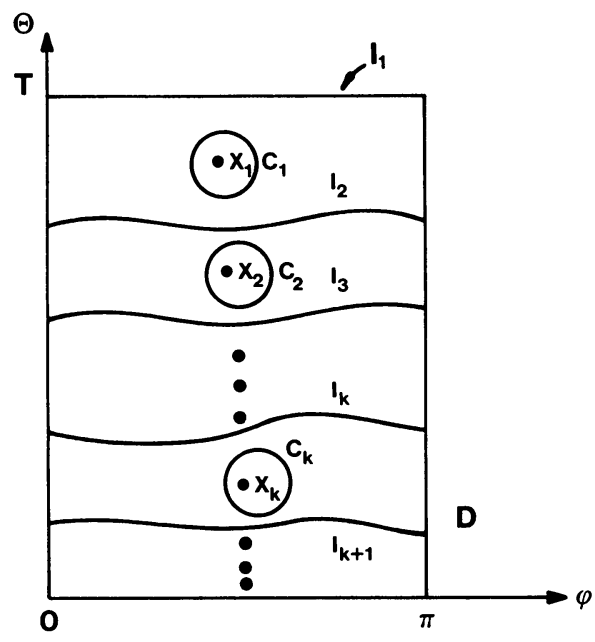

FIGURE 4

ProOF. Let $Z_{k}=\{x \in X: h(x)=k\}$. From Proposition 2.5, there exists $\theta_{k}>0$ such that if $x=(\varphi, \theta) \in Z_{k}$, then $\theta>\theta_{k}$. By compactness and continuous dependence, it follows that for each $k$ there exists $\delta_{k}>0$ such that $\delta(x)>\delta_{k}$ for each $x \in Z_{k}$.

Suppose that $Z_{k}=Z_{k_{1}} \cup Z_{k_{2}} \cup \cdots \cup Z_{k n}$ where

(a) if $x_{1} \in Z_{k i}$ and $x_{2} \in Z_{k j}$ for $i \neq j$, then $\left\|x_{1}-x_{2}\right\|>\delta_{k}$,

(b) if $x_{1} \in Z_{k i}$ and $x_{2} \in Z_{k i}$, then there exists $d_{1}=x_{1}, d_{2}, \ldots, d_{n}=x_{2}$ such that each $d_{i} \in Z_{k i}$ and $\left\|d_{i}-d_{i+1}\right\|<\delta_{k}$ for each $i$.

To complete the proof we must show that there exist mutually disjoint closed curves $C_{k j} \subset Y$ such that for each $k, j$,

(a) $Z_{k j}$ lies in the disc bounded by $C_{k j}$,

(b) if $y \in C_{k j}$, then there exists $x \in Z_{k j}$ such that $\|x-y\|<\delta_{k}$. The proof of this last result is straightforward, so we do not give the details.

REMARK. (1) It is possible that one of the $C_{k}$ 's lies inside another. We only consider those $C_{k}$ 's which do not lie inside another one. We order the $C_{k}$ 's so that if $i<j$, then

$$
\sup \left\{\theta:(\varphi, \theta) \in C_{i}\right\} \geq \sup \left\{\theta:(\varphi, \theta) \in C_{j}\right\}
$$

(2) The constants $\delta_{k}$ appearing in the proposition will be chosen later.

In Figure 4 we illustrate the $X_{k}$ 's and $C_{k}$ 's. We assume, for convenience, in Figure 4 that $\varphi_{1}(\theta)=0$ and $\varphi_{2}(\theta)=\pi$ for each $\theta \in[0, T]$. The $I_{k}$ shown in Figure 4 will be defined in the next section.

\section{Reduction to the algebraic problem.}

A. The basic set up. Let $\mathcal{G}$ be the set of continuous functions $g:[0,1] \rightarrow Y$ described in $\S 2$. Let $\left\{I_{k}\right\}$ be a sequence of functions in $\mathcal{G}$ such that

(a) $I_{1}(s)=\left((1-s) \varphi_{1}(T)+s \varphi_{2}(T), T\right)$ for $s \in[0,1]$.

(b) $I_{k}(s)$ separates $D$ into two regions. The "top" half contains $C_{1}, \ldots, C_{k-1}$, and the "bottom half" contains $C_{k}, C_{k+1}, \ldots$

(c) $\sup \left\{\theta: I_{k}(s)=(\varphi, \theta)\right.$ for some $\left.s\right\}<\sup \left\{\theta:(\varphi, \theta) \in C_{k}\right\}+1 / k$. 
To each $I_{k}$ there corresponds the algebraic objects $\Gamma^{*}\left(I_{k}\right)$ and $\Gamma\left(I_{k}\right)$. These were defined in $\S 2$. Let $\Gamma_{k}=\Gamma\left(I_{k}\right)$ and $h_{k}=h(x)$ for any $x \in X_{k}$. Because $x_{1}, x_{2} \in X_{k}$ implies $h\left(x_{1}\right)=h\left(x_{2}\right)$, it follows that $h_{k}$ is well defined.

Theorem 1 is equivalent to proving

Proposition 4A.1. For each positive integer $K$ there exists $k$ such that either $h_{k}=K$ or $h_{k}=K+1$.

In this section we show that the sequence $\left\{\Gamma_{k}\right\}$ satisfies (a)-(c) of Proposition 1F.1. Moreover, $\Gamma_{A}$ can be chosen so that $h_{k}=\omega\left(\Gamma_{A}\right)$. The proof of Proposition 4A.1 then follows from Proposition 1E.1. Actually, as we discussed in the introduction, we do not verify (b) of Proposition 1 F.1 in this paper.

B. $\Gamma_{1}=\beta \gamma^{-1}$. We now verify (1E.4a); that is, $\Gamma_{1}=\beta \gamma^{-1}$. This is proved by continuing the given system to one in which it is possible to compute $\Gamma_{1}$ directly. We begin by defining the target system.

Let $g: R \rightarrow R$ be any function which satisfies

(a) $g \in C^{2}(\mathbf{R}), \lim _{|u| \rightarrow \infty} g(u)=-\infty$.

(b) $g$ has exactly three local maxima. These are at $A_{1}, B_{1}$, and $C_{1}$.

(c) $g\left(A_{1}\right)<g\left(B_{1}\right)<g\left(C_{1}\right)$.

(d) $g$ has two local minima. These are at $D_{1}$ and $E_{1}$.

Let $F_{1}\left(U_{1}, U_{2}\right)=g\left(U_{1}\right)-U_{2}^{2}$. Choose $K>\max \left\{\left|B_{1}\right|,\left|C_{1}\right|\right\}$, and let $N_{1}^{1}=$ $\left\{\left(U_{1}, U_{2}\right):\left|U_{1}\right| \leq K,\left|U_{2}\right| \leq K\right\}$. One easily checks that if $U \in \partial N_{1}^{1}$, then $U$. $\nabla F_{1}(U)<0$.

Let $F_{\eta}\left(U_{1}, U_{2}\right), 0 \leq \eta \leq 1$, be a one parameter family of functions which satisfy for each $\eta$,

(a) $F_{\eta} \in C^{2}\left(\mathbf{R}^{2}\right)$.

(b) $F_{\eta}(U)$ has at least three nondegenerate local maxima. These are at $A^{\eta}=$ $(0,0), B^{\eta}$, and $C^{\eta}$.

(c) $F_{\eta}(U)$ has at least two saddles. These are at $D^{\eta}$ and $E^{\eta}$.

(d) $B_{1}^{\eta}<D_{1}^{\eta}<A_{1}^{\eta}<E_{1}^{\eta}<C_{1}^{\eta}$ and $F_{\eta}\left(A^{\eta}\right)<F_{\eta}\left(B^{\eta}\right)<F_{\eta}\left(C^{\eta}\right)$.

(e) $F_{\eta}(U)$ is a continuous function of $\eta$ for each $U \in \mathbf{R}^{2}$.

(f) $F_{0}(U)=F(U)$ for each $U \in \mathbf{R}^{2}$.

(g) There exists convex sets $N_{1}^{\eta} \in \mathbf{R}^{2}$ which depend continuously on $\eta$ such that, for each $\eta, U \cdot \nabla F(U)<0$ for $U \in \partial N_{1}^{\eta}$.

(h) If $U_{1}=D_{1}^{\eta}$ or $E_{1}^{\eta}$, then $\partial F_{\eta} / \partial U_{1}=0$.

Now consider the system

$$
U^{\prime}=V, \quad V^{\prime}=\theta V-\nabla F_{\eta}(U) .
$$

For each $\eta, \theta$ let $W_{\eta}^{\theta}$ be the unstable manifold at $\left(A^{\eta}, 0\right)$ for $(4 \mathrm{~B} .1 \eta)$. We now repeat our previous constructions for the new systems (4B.1 $\eta)$. Here we outline the details.

Choose $\bar{V}_{\eta}$ so that if $\left(U^{\eta}(z), V^{\eta}(z)\right)$ is a bounded solution of $(4 \mathrm{~B} .1 \eta)$, then $\left\|V^{\eta}(z)\right\|<\bar{V}_{\eta}$. We may assume that $\bar{V}_{\eta}$ is independent of $\eta$. Assume that $\bar{V}$ satisfies $\bar{V}>\bar{V}_{\eta}$ for each $\eta$. For $\eta \in[0,1]$ let

$$
\begin{aligned}
& N_{2}^{\eta}=\left\{(U, V): U \in N_{1}^{\eta},\|V\| \leq \bar{V}\right\}, \\
& P_{D}^{\eta}=\left\{(U, V): U_{1}=D_{1}^{\eta}, V_{1}=0\right\} \\
& P_{E}^{\eta}=\left\{(U, V): U_{1}=E_{1}^{\eta}, V_{1}=0\right\}, \\
& N^{\eta}=N_{2}^{\eta} \backslash\left(P_{D}^{\eta} \cup P_{E}^{\eta}\right) .
\end{aligned}
$$




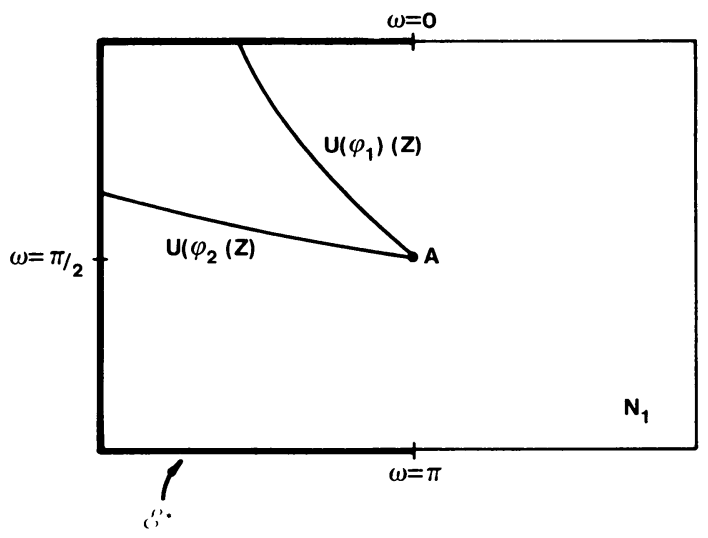

FIGURE 5

Choose $T$ so large that no nonconstant, bounded solutions of (4B.1 $\eta)$ exist for any $\eta, \theta>T$. As before, we assume that $F^{\eta}\left(C^{\eta}\right)$ is so big that if $(U(z), V(z)) \in W_{\eta}^{\theta}$ with $\theta<T$, then $U(z) \neq C$ for all $z$.

As in $\S 2$ we parametrize the trajectories in $W_{\eta}^{\theta}, 0 \leq \theta \leq T$, by the rectangle

$$
D_{1}^{\eta}=\{(\varphi, \theta): 0 \leq \varphi \leq 2 \pi, 0<\theta \leq T\} \text {. }
$$

As in the remarks following Lemma 2.6 we are only interested in half of $D_{1}^{\eta}$. Let $X_{2}^{\eta}=\left\{U: D_{1}^{\eta} \leq U \leq E_{1}^{\eta}\right\}$. Choose $q_{1}(\eta)$ and $q_{2}(\eta)$ to be points on, respectively, the top and bottom sides of $X_{2}^{\eta}$, similar to what is shown in Figure 2. As in Lemma 2.6 there exists continuous functions $\varphi_{1}(\eta, \theta)$ and $\varphi_{2}(\eta, \theta)$ such that for each $\eta, \theta, i=1,2, U^{\eta}\left(\varphi_{i}(\eta, \theta), \theta\right)(z)$ leaves $X_{2}^{\eta}$ through $q_{i}(\eta)$. We may choose $\varphi_{1}(\eta, \theta)$ and $\varphi_{2}(\eta, \theta)$ to depend continuously on $\eta$ and $\theta$. Let

$$
\begin{aligned}
& D^{\eta}=\left\{(\varphi, \theta) \in D_{1}^{\eta}: \varphi_{1}(\eta, \theta) \leq \theta \leq \varphi_{2}(\eta, \theta)\right\}, \\
& X^{\eta}=\left\{(\varphi, \theta) \in D_{1}^{\eta}: \gamma^{\eta}(\varphi, \theta)(z) \rightarrow\left(B_{\eta}, 0\right) \text { as } z \rightarrow \infty\right\}, \\
& Y^{\eta}=D^{\eta} \backslash X^{\eta}
\end{aligned}
$$

Let

$$
\mathcal{E}^{\eta}=\left\{(U, V) \in \partial N^{\eta}:\|V\|<\bar{V}\right\} .
$$

We may choose $\bar{V}$ so large that if $y \in Y^{\eta}$, then $\gamma^{\eta}(y)(z)$ leaves $N^{\eta}$ through $\mathcal{E}^{\eta}$. We now have a continuous mapping $\Lambda^{\eta}: Y^{\eta} \rightarrow \mathcal{E}^{\eta}$ such that $\Lambda^{\eta}(y)$ is equal to the place where $\gamma^{\eta}(y)(z)$ leaves $N^{\eta}$.

Define $g^{\eta}: I \rightarrow Y^{\eta}$ by

$$
g^{\eta}(s)=\left((1-s) \varphi_{1}(\eta, T)+s \varphi_{2}(\eta, T), T\right) .
$$

We can then define the algebraic object $\Gamma^{\eta}$ corresponding to $\Lambda^{\eta} \circ g^{\eta}$. Of course, $\Gamma^{0}=\Gamma_{1}$. To prove that $\Gamma_{1}=\beta \gamma^{-1}$ we show that $\Gamma^{1}=\beta \gamma^{-1}$ and that $\Gamma^{\eta}$ is independent of $\eta$.

We now show that $\Gamma^{1}=\beta \gamma^{-1}$. Note that when $\eta=1,(4 \mathrm{~B} .1 \eta)$ splits into the product of the two, two dimensional systems:

$$
U_{1}^{\prime}=V_{1}, \quad V_{1}^{\prime}=\theta V_{1}-h^{\prime}\left(U_{1}\right)
$$


and

$$
U_{2}^{\prime}=V_{2}, \quad V_{2}^{\prime}=\theta V_{2}-2 V_{2} \text {. }
$$

As in the remarks following Proposition 2.3, the trajectories in $W_{1}^{\theta}$ are parametrized by the points on the circle $A^{\delta}=\{U:\|U-A\|=\delta\}$ for $\delta$ sufficiently small. The points in $A^{\delta}$ are parametrized by the angle $\varphi$, where $\varphi=0$ is chosen as in Figure 2. Let

$$
\gamma^{1}(\varphi)(z)=(U(\varphi)(z), V(\varphi)(z))
$$

be the trajectory in $W_{1}^{\theta}$ corresponding to the angle $\varphi$. From (4B.2) it follows that when $\varphi=0$ or $\pi$,

$$
U_{1}(\varphi)(z)=A_{1} \quad \text { and } \quad V_{1}(\varphi)=0 \quad \text { for all } z
$$

Moreover, $V_{2}(0)(z)>0$ and $V_{2}(\pi)(z)<0$ for all $z$. We also have that $U_{2}(\pi / 2)(z)=$ $V_{2}(\pi / 2)(z)=0$ for all $z$. Moreover, $V_{1}(\pi / 2)(z)<0$ for all $z$. This implies that $\varphi_{1}(1, T)=0$ and $\varphi_{2}(1, T)=\pi$, if $q_{1}(1)$ and $q_{2}(1)$ are chosen appropriately.

Let $\mathcal{E}^{*}=\left\{U:(U, V) \in \mathcal{E}^{1}, U_{1} \leq A_{1}\right\}$. Because $U_{1}^{\prime}(\varphi)(z) \leq 0$ for $\varphi \in[0, \pi]$ it follows that if $\varphi \in[0, \pi]$, then $U(\varphi)(z)$ leaves $N_{1}^{1}$ through $\mathcal{E}^{*}$. We parametrize $\mathcal{E}^{*}$ by the angle $\omega$ shown in Figure 5. We then have the continuous map $\Phi:[0, \pi] \rightarrow[0, \pi]$ where $\Phi(\varphi)$ is the value of $\omega$ corresponding to the place where $U(\varphi)(z)$ leaves $N_{1}^{1}$.

LEMMA 4B.1. $\Phi$ is an increasing function of $\varphi$.

ProOF. We prove the lemma by showing that if $\varphi_{1} \neq \varphi_{2}$, then $U\left(\varphi_{1}\right)\left(z_{1}\right) \neq$ $U\left(\varphi_{2}\right)\left(z_{2}\right)$ for any $z_{1}$ and $z_{2}$. The picture is then like what is shown in Figure 5 .

Suppose that there exists $\varphi_{1}, \varphi_{2}, z_{1}, z_{2}$ such that $U\left(\varphi_{1}\right)\left(z_{1}\right)=U\left(\varphi_{2}\right)\left(z_{2}\right)$. Assume that $\varphi_{1}<\varphi_{2}$. Clearly, either $\varphi_{1}<\varphi_{2}<\pi / 2$ or $\pi / 2<\varphi_{1}<\varphi_{2}$. We assume that $\varphi_{1}<\varphi_{2}<\pi / 2$. The other case is similar. Since (4B.2) is a product system, there exists $\xi, \eta$ such that, up to translation,

$$
U\left(\varphi_{1}\right)(z)=\left(U_{1}(\pi / 2)(z), U_{2}(0)(z-\xi)\right)
$$

and

Therefore,

$$
U\left(\varphi_{2}\right)(z)=\left(U_{1}(\pi / 2)(z), U_{2}(0)(z-\eta)\right)
$$

$$
U_{1}(\pi / 2)\left(z_{1}\right)=U_{1}(\pi / 2)\left(z_{2}\right)
$$

and

$$
U_{2}(0)\left(z_{1}-\xi\right)=U_{2}(0)\left(z_{2}-\eta\right) .
$$

Since $U_{1}(\pi / 2)(z)$ and $U_{2}(0)(z)$ are monotonic it follows that $z_{1}=z_{2}$ and $z_{1}-\xi=$ $z_{2}-\eta$. Hence, $\xi=\eta$. If $\varphi_{1} \neq \varphi_{2}$, then this is clearly impossible, thus proving the lemma.

LEMMA 4B.2. $\Gamma^{1}=\beta \gamma^{-1}$.

PROOF. A $g^{1}$-partition is $\{0, \pi / 2, \pi\}$. In the notation of $\S 2, \Lambda^{1} \circ g(0) \in \mathcal{E}_{2}$, $\Lambda^{1} \circ g(\pi / 2) \in \mathcal{E}_{3}$, and $\Lambda^{1} \circ g(\pi) \in \mathcal{E}_{4}$. From the definitions, and the preceding result we find that $\Lambda^{1} \circ g(s) \in l_{\beta}^{+}$for some $s \in(0, \pi / 2)$, and $\Lambda^{1} \circ g(s) \in l_{\gamma}^{-}$for some $s \in(\pi / 2, \pi)$. The result now follows from Table 1 in $\S 2$.

It remains to prove that $\Gamma^{\eta}=\beta \gamma^{-1}$ for each $\eta$; that is, $\Gamma^{\eta}$ depends continuously on $\eta$. We only outline the proof. Let $J=\left\{\eta: \Gamma^{\eta}=\beta \gamma^{-1}\right\}$. We have shown that 


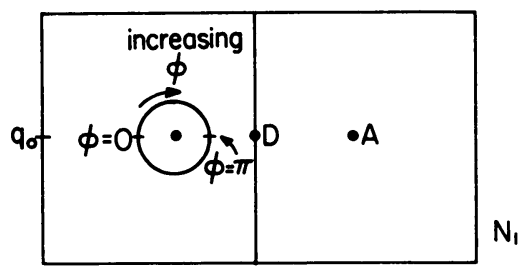

FIGURE 6

$J$ is nonempty. $J$ is open because everything depends continuously on $\eta$. The only thing that could stop $J$ from being closed is if, for some $\eta, \gamma^{\eta}(d)(z)$ does not leave $N^{\eta}$ for some $d=(\varphi, T)$. However, $T$ was chosen so that if $d=(\varphi, T)$, then $d \in Y^{\eta}$. If $d \in Y^{\eta}$, then we know that $\gamma^{\eta}(d)(z)$ must leave $N^{\eta}$. This completes the proof.

C. The unstable manifold at $(B, O)$. In this section we show that $W_{B}^{\theta}$, the unstable manifold at $(B, 0)$ for a given $\theta>0$, generates elements of $F_{4}$ in a way similar to $W_{A}^{\theta}$. The construction is a little different from before, so we go through it in some detail.

As in $\S 2$ we may assume that $F(C)$ is so big that if $(U(z), V(z)) \in W_{B}^{\theta}$, then $U(z) \neq C$ for all $z$ and $\lim _{z \rightarrow \infty} U(z) \neq C$. Together with (2.2) this implies that each nontrivial trajectory in $W_{B}^{\theta}, \theta>0$, must leave $N$. We choose $\bar{V}$ so large that each nontrivial trajectory in $W_{B}^{\theta}$ leaves $N$ through $\mathcal{E}$.

As with $W_{A}^{\theta}$ we parametrize the nontrivial trajectories in $W_{B}^{\theta}$; however, now we parametrize the nontrivial trajectories in $W_{B}^{\theta}$ by the points in a cylinder. Let $B_{\delta}=\{U:\|U-B\|=\delta\}$.

The key to the parametrization is the following

Proposition 4C.1. If $\delta$ is sufficiently small, then (a) for each $U \in B_{\delta}$ there exists a unique $V$ such that $(U, V) \in W_{B}^{\theta}$, and (b) if $(U(z), V(z))$ is a nontrivial trajectory in $W_{B}^{\theta}$, then there exists a unique $z_{0}$ such that $U\left(z_{0}\right) \in B_{\delta}$.

This is proved by linearizing the system at $(B, 0)$, proving the result for the linear system, and then using the fact that $W_{B}^{\theta}$ is tangent to the unstable manifold of the linear system at $(B, 0)$.

Now parametrize the points in $B_{\delta}$ by the angle $\varphi$ as shown in Figure 6. That is, $\varphi=0$ corresponds to the point $\left(B_{1}-\delta, B_{2}\right)$ and $\varphi$ increases in the clockwise direction.

In the construction of $\Gamma_{k}^{*}$ and $\Gamma_{k}$ it was necessary to have the "base points" $q_{1}$ and $q_{2}$ shown in Figure 2. We need a base point now. Throughout this section we assume that

is the trajectory in $W_{B}^{\theta}$ corresponding to the angle $\varphi$.

$$
\gamma_{B}(\varphi, \theta)(z)=\left(U_{B}(\varphi, \theta)(z), V_{B}(\varphi, \theta)(z)\right)
$$

LEMMA 4C.2. Fix $\theta$ and choose $q \in \partial X_{3}$. Then there exists $\varphi_{3}(\theta)$ such that $U_{B}\left(\varphi_{3}(\theta), \theta\right)(z)$ leaves $X_{3}$ at $q$. Moreover, $\varphi_{3}(\theta)$ can be chosen to depend continuously on $\theta$.

The proof of this result is similar to the proof of Lemma 2.6 so we do not give the details. Let $q_{0}$ be the point shown in Figure 6. That is, if $q_{0}=\left(q^{1}, q^{2}\right)$, then $q^{2}=B_{2}$. For each $\theta$, let $\varphi_{3}(\theta)$ be such that $U_{B}\left(\varphi_{3}(\theta), \theta\right)$ leaves $X_{3}$ at $q_{0}$. 


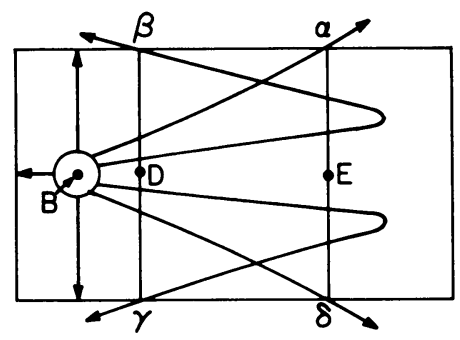

FiguRE 7

Let $D_{B}$ be the cylinder

$$
D_{B}=\{(\varphi, \theta): \varphi \in[0,2 \pi], 0<\theta \leq T\}
$$

where we identify $\varphi=0$ with $\varphi=2 \pi$. Recall that if $(\varphi, \theta) \in D_{B}$, then $\gamma_{B}(\varphi, \theta)(z)$ must leave $N$ through $\mathcal{E}$. Define $\Phi: D_{B} \rightarrow N$ where $\Phi(\varphi, \theta)$ is equal to the place where $\gamma_{B}(\varphi, \theta)(z)$ leaves $N$.

Let $\mathcal{G}_{B}$ equal the set of continuous functions from $[0,1]$ to $D_{B}$ such that $g(0)=$ $g(1) \in\left\{(\varphi, \theta): \varphi=\varphi_{3}(\theta)\right\}$. For $g \in \mathcal{G}_{B}, \Phi \circ g$ defines a curve in $\mathcal{E}$. As in $\S 2$ we can assign to $\Phi \circ g$ an element $\Lambda(g)$ of $F_{4}$. We do not go through the details because the construction is the same as in $\S 2$.

The key result of this section is

PROPOSITION 4C.3. If $g \in \mathcal{G}_{B}$, then there exists an integer $r$ such that

$$
\Lambda(g)=\left(\alpha \beta \gamma^{-1} \delta^{-1}\right)^{r} .
$$

ProOF. Assume, for convenience, that $\varphi_{3}(\theta)=0$ for each $\theta$. Define $g_{1} \in \mathcal{G}_{B}$ by

$$
g_{1}(s)=(2 \pi s, T) .
$$

Let $g_{1}^{*}: \mathbf{R} \rightarrow \nu_{B}$ be the lift of $g_{1}$ to $R$. That is, if $s \in \mathbf{R}$ and $n \leq s<n+1$, then $g_{1}^{*}(s)=g_{1}(s-n)$. For each integer $r$ define $g_{r}(s): I \rightarrow C_{\delta}$ by $g_{r}(s)=g_{1}^{*}(r s)$. Then $g_{r}(I)$ is a curve which winds around $D_{B} r$ times.

Now assume that $g \in \mathcal{G}_{B}$. Clearly there exists an integer $r$ such that $g$ is homotopic to $g_{r}$ relative to $D_{B}$. It is a fact (which we do not prove here) that if $g$ is homotopic to $g_{r}$ relative to $D_{B}$, then $\Lambda(g)=\Lambda\left(g_{r}\right)$. Clearly

$$
\Lambda\left(g_{r}\right)=\left[\Lambda\left(g_{1}\right)\right]^{r} \text {. }
$$

To complete the proof of the proposition it remains to prove that

$$
\Lambda\left(g_{1}\right)=\alpha \beta \gamma^{-1} \delta^{-1}
$$

The proof of (4C.3) is very similar to the proof that $\Gamma_{1}=\beta \gamma^{-1}$. We continue the given system (1A.4) to the product system (4B.2). One can compute $\Lambda$ for the product system explicitly. One then proves that $\Lambda$ is invariant under the continuation. We do not give the details, however in Figure 7 we show the projection of various trajectories in $W_{B}^{\theta}$ for (4B.2) with $\theta$ very large.

REMARK. Suppose that $g(s)$ is a continuous map from $[0,1]$ to $D_{B}$, but $g(s) \notin$ $\left\{(\varphi, \theta): \varphi=\varphi_{3}(\theta)\right\}$ for all $s$. Then $g \notin D_{B}$, but $\Gamma \circ g$ still defines a curve in $\mathcal{E}$ to which we can define an element of $F_{4}$. It is not hard to verify that because $g$ is 
homotopic to the constant function relative to $D_{B}$ we have that $\Lambda \circ g$ is the trivial element of $F_{4}$. Hence, (4C.1) still holds, except now, $r=0$.

We will need a result a bit more general than Proposition 4C.3. We must "fatten" $B_{\delta}$ a little and consider trajectories close to the trajectories in $W_{B}^{\theta}$. Let $\delta$ be as in Proposition 4C.1 and $\lambda=\delta / 2$. For each $\varphi \in[0,2 \pi], \theta \in(0, T]$ there is a unique point $\left(U_{B}(\varphi, \theta), V_{B}(\varphi, \theta)\right) \in W_{B}^{\theta}$ such that $\left\|U_{B}(\varphi, \theta)-B\right\|=\delta$. Let

$$
\begin{aligned}
C & =\bigcup_{(\varphi, \theta) \in D_{B}}\left(U_{B}(\varphi, \theta), V_{B}(\varphi, \theta)\right), \\
C_{\lambda} & =\{(U, V):\|(U, V)-C\| \leq \lambda\}, \\
P & =\left\{(U, V) \in C_{\lambda}: U=U\left(\varphi_{3}(\theta), \theta\right) \text { for some } \theta\right\} .
\end{aligned}
$$

Recall that for each $\theta, U\left(\varphi_{3}(\theta), \theta\right)(z)$ leaves $X_{3}$ at the point $q_{0}$. By making $\lambda$ smaller, if necessary, we may assume that if $p \in P$ and $\gamma(p)(z)$ is the solution of (1A.4) with $\gamma(p)(0)=p$, then $U(p)(z)$ leaves $X_{3}$ through its left side.

Now let $G^{*}$ be the set of all continuous functions $g:[0,1] \rightarrow C_{\lambda}$ such that $g(0)=$ $g(1) \in P$.

Making $\lambda$ still smaller, if necessary, we may assume that if $p \in C_{\lambda}$, then $\gamma(p)(z)$ leaves $N$ through $\mathcal{E}$. Let $\Phi^{*}(p)$ equal the place where $\gamma(p)(z)$ leaves $N$. If $g \in G^{*}$, then $\Phi^{*} \circ g:[0,1] \rightarrow \mathcal{E}$ defines a continuous, closed curve in $\mathcal{E}$. We can define $\Lambda(g)$, an element of $F_{4}$, as before. From Proposition 4C.3 it follows that if $\lambda$ is sufficiently small, then we have

PROPOSITION 4C.4. If $g \in G^{*}$, then $\Lambda(g)=\left(\alpha \beta \gamma^{-1} \delta^{-1}\right)^{r}$ for some integer $r$.

REMARK. As with the remark following Proposition 4C.3, if $g:[0,1] \rightarrow C_{\lambda}$ and $g(s) \notin P$ for all $s$, then we can still define $\Lambda(g)$. In this case, (4C.1) holds with $r=0$.

D. $\Gamma_{k} \rightarrow \Gamma_{k+1}$. We now verify that $\Gamma_{k}$ and $\Gamma_{k+1}$ satisfy (c) of Proposition $1 \mathrm{~F} .1$. That is, we prove

Proposition 4D.1. Let $k$ be a positive integer. Then there exists $\Gamma_{A} \in F_{4}$, $\Gamma_{B} \in F_{4}$, and an integer $r$ such that

(a) $\Gamma_{A} \Gamma_{B} \in \Gamma_{k}^{*}$ and

(b) $\Gamma_{A}\left(\alpha \beta \gamma^{-1} \delta^{-1}\right)^{r} \Gamma_{B} \in \Gamma_{k+1}^{*}$.

REMARK. $r$ may be positive, negative, or zero.

PROOF. The proof of this result is split into a number of steps. To begin with recall how $\Gamma_{k}$ was defined. We started with a map $I_{k} \in \mathcal{G}$ which satisfies (4A.1). From $I_{k}$ we defined $\Gamma_{k}^{*}\left(I_{k}\right)$, and making cancellations we obtained $\Gamma_{k} . \Gamma_{k}$ does not depend on $I_{k}$ in the sense that any function $I_{k}^{\prime} \in \mathcal{G}$ which is homotopic to $I_{k}$ relative to $Y$ yields the same $\Gamma_{k}$. We shall now be more careful about how we choose $I_{k}$.

In addition to (4A.1) we require that $I_{k}\left(s_{0}\right) \in C_{k}$ for one and only one $s_{0} \in[0,1]$. That is, $I_{k}(s)$ touches $C_{k}$ at one point which we denote by $p_{k}$. We shall describe how to choose $p_{k}$ shortly.

First we describe how to define $I_{k+1}$. It will be the union of three curves. These are

$$
\begin{aligned}
& \text { (a) } I_{k}(s) \text { for } 0 \leq s \leq s_{0} \text {, } \\
& \text { (b) } C_{k} \text {, } \\
& \text { (c) } I_{k}(s) \text { for } s_{0} \leq s \leq 1 \text {. }
\end{aligned}
$$


More specifically, $C_{k}$ is homeomorphic to the unit circle. Say $C_{k}$ is given by the $\operatorname{map} L: I \rightarrow Y$ where $L(0)=L(1)=p_{k}$. Let

$$
I_{k+1}(s)= \begin{cases}I_{k}\left(3 s_{0} s\right) & \text { for } 0 \leq s \leq \frac{1}{3} \\ L(3 s-1) & \text { for } \frac{1}{3} \leq s \leq \frac{2}{3} \\ I_{k}\left(3\left(1-s_{0}\right) s-2+3 s_{0}\right) & \text { for } \frac{2}{3} \leq s \leq 1\end{cases}
$$

Hence, to obtain $I_{k+1}$ from $I_{k}$ we attach the loop $C_{k}$.

Let $\eta=\left\{\eta_{1}, \ldots, \eta_{K}\right\}$ be an $I_{k}$-partition. Choose $j$ so that $\eta_{j} \leq s_{0} \leq \eta_{j+1}$. In the notation of $\S 2$, we assume that $\eta_{j+1}-\eta_{j}$ is so small that $e_{j+1}=0$. There is no problem with this assumption because any refinement of an $I_{k}$-partition is also an $I_{k}$-partition. Let $\Gamma_{A}=\lambda_{1}^{e_{1}} \cdots \lambda_{j}^{e_{j}}$ and $\Gamma_{B}=\lambda_{j+1}^{e_{1}} \cdots \lambda_{K}^{e_{K}}$. From the definitions we have that $\Gamma^{*}\left(I_{k}, \eta\right)=\Gamma_{A} \Gamma_{B}$. Hence, $\Gamma_{A} \Gamma_{B} \in \Gamma_{k}^{*}$.

We now compute $\Gamma^{*}\left(I_{k+1}\right)$. We must find an $I_{k+1}$-partition, $\eta^{*}$. But we are two-thirds of the way done because $I_{k+1}$ consists of three pieces and we have a partition of two of them. To (4D.1a) corresponds the sequence $\Gamma_{A}$, and to (4D.1b) corresponds $\Gamma_{B}$. Suppose that the, yet to be computed, sequence corresponding to (4D.1b) is denoted by $\Gamma^{*}\left(C_{k}\right)$. Then,

$$
\Gamma^{*}\left(I_{k+1}, \eta^{*}\right)=\Gamma_{A}\left[\Gamma^{*}\left(C_{k}\right)\right] \Gamma_{B} .
$$

Therefore, it remains to prove that

$$
\Gamma^{*}\left(C_{k}\right)=\left(\alpha \beta \gamma^{-1} \delta^{-1}\right)^{r}
$$

for some integer $r$.

Choose $y \in C_{k}$. Then from Proposition 3.4 there exists $x \in X_{k}$ such that $\|x-y\|<\delta_{k}$ where $\delta_{k}$ has yet to be determined. By making $\delta_{k}$ small, $\gamma(y)(z)$ will lie as close to $\gamma(x)(z)$ as we please, for as long as we please. Since $\lim _{z \rightarrow \infty} \gamma(x)(z)=$ $(B, 0)$ it follows that $\gamma(y)(z)$ passes arbitrarily close to $(B, 0)$. As it leaves a small neighborhood of $(B, O), \gamma(y)(z)$ must lie very close to $W_{B}^{\theta}$. Hence, by choosing $\delta_{k}$ small we have that $\gamma(y)(z) \in C_{\lambda}$ for some $z$. Recall that $C_{\lambda}$ was defined in the previous section. We now have a mapping

$$
G: C_{k} \rightarrow C_{\lambda}
$$

given by $G(y)=\gamma(y)(z)$ where $z$ is chosen so that $\gamma(y)(z) \in C_{\lambda}$. We may certainly choose $z$ so that $G(y)$ is continuous.

We now consider two cases. Let $P$ be as in the preceding section. If $G\left(y_{0}\right) \in P$, for some $y_{0}$, let $p_{k}=y_{0}$. Then $G \circ L\left(C_{k}\right) \in G^{*}$. It follows from Proposition 4 C.4 that

$$
\Lambda\left(G \circ L\left(C_{k}\right)\right)=\left(\alpha \beta \gamma^{-1} \delta^{-1}\right)^{r}
$$

from some integer $r$. However, from the definitions, $\Lambda\left(G \circ L\left(C_{k}\right)\right)=\Gamma^{*}\left(C_{k}\right)$ so the proof of the proposition is complete.

It remains to consider the case $G(y) \notin P$ for all $y$. Let $p_{k}$ be arbitrary now. We still have that $G\left(C_{k}\right)$ is a continuous curve in $C_{\lambda}$. The remark following Proposition 4 C. 4 implies that $\Lambda\left(G \circ L\left(C_{k}\right)\right)=\left(\alpha \beta \gamma^{-1} \delta^{-1}\right)^{r}$ for $r=0$. Because $\Lambda\left(G \circ L\left(C_{k}\right)\right)=$ $\Gamma^{*}\left(C_{k}\right)$ we are done.

E. Completion. There are two steps remaining in the reduction of the proof of the theorem to the algebraic problem, Proposition 4E.1. We must prove

(a) (b) of Proposition (1E.1),

(b) $h_{k}=\omega\left(\Gamma_{A}\right)$. 
As we mention in the introduction, we are not able to even define $\left\|\Gamma_{k}\right\|$ in this paper, let alone prove (4E.1a). We must first develop quite a bit of the algebraic theory before we can define $\left\|\Gamma_{k}\right\|$. We point out however that in [1, Proposition 5A.1] we prove the weaker result that for any integer $M$ there exists $K$ such that if $k>K$, then $\left|\Gamma_{k}\right|>M$.

It remains to verify $(4 \mathrm{E} .1 \mathrm{~b})$. Recall the point $p_{k}$ defined in the preceding section. From Proposition 2.6 and the definition of $\Gamma_{A}$ we conclude that $h\left(p_{k}\right)=\omega\left(\Gamma_{A}\right)$. So we are done if we can show that $h\left(p_{k}\right)=h_{k}$.

Choose $x \in X_{k}$. Now $U(x)(z)$ winds back and forth $h_{k}$ times, and then enters $B^{\delta}=\{U:\|B-U\| \leq \delta\}$ where $\delta$ is as in Proposition 4C.1.

By continuous dependence, if $\delta_{0}$ is sufficiently small, then $U\left(p_{k}\right)(z)$ will wind back and forth $h_{k}$ times and then enter $B^{\delta}$. By assumption, $\gamma\left(p_{k}\right)(z) \in \mathcal{P}$ for some $z$. From the definition of $P$, after $\gamma\left(p_{k}\right)(z)$ leaves $B^{\delta}$ it proceeds to leave $X_{3}$ through its left side. Hence, after entering $B^{\delta}, U\left(p_{k}\right)(z)$ does not wind around any more, thus proving the desired result.

REMARK. We have yet to explain why Remark 2 in $\S 1 \mathrm{D}$ is true. Recall that in our definition of $D$ in $\S 2$ we only considered values of $(\varphi, \theta)$ for which $\varphi_{1}(\theta) \leq \varphi \leq$ $\varphi_{2}(\theta)$. We proved that for each positive integer $K$ there exists a traveling wave solution with winding number $K$ or $K+1$. Moreover, the traveling wave solution corresponds to a value of $(\varphi, \theta)$ with $\varphi_{1}(\theta) \leq \varphi \leq \varphi_{2}(\theta)$. We could have just as easily assumed that $\varphi \notin\left[\varphi_{1}(\theta), \varphi_{2}(\theta)\right]$. This would have given us another traveling wave solution with winding number $K$ or $K+1$.

\section{REFERENCES}

1. C. Conley, Isolated invariant sets and the Morse index, CBMS Regional Conf. Ser. in Math., no. 38, Amer. Math. Soc., Providence, R. I., 1978.

2. R. Franzosa, Index filtrations and connection matrices for partially ordered Morse decompositions, Ph.D. dissertation, Univ. of Wisconsin, Madison, 1984.

3. K. Mischaikow, Classification of traveling wave solutions of reaction-diffusion systems, Brown Univ., LCDS \#86-5, 1985.

4. J. Reineck, Connecting orbits in one parameter families of flow, preprint.

5. D. Terman, Directed graphs and traveling waves, Trans. Amer. Math. Soc. 289 (1985), 809-847.

6. , Infinitely many traveling wave solutions of a gradient system, Trans. Amer. Math. Soc. 301 (1987), 537-556.

7. __ Traveling wave solutions of a gradient system: Solutions with a prescribed winding number. II, Trans. Amer. Math. Soc. (to appear).

8. _ Infinitely many radial solutions of an elliptic system, Ann. Inst. H. Poincaré, Analyse Non Lineaire 4 (1987), 549-604.

9. __ Radial solutions of an elliptic system: Solutions with a prescribed winding number, Houston J. Math. (to appear).

Department of Mathematics, Michigan State University, East Lansing, MICHIGAN 48824

Current address: Department of Mathematics, Ohio State University, Columbus, Ohio 43210 